\title{
Reseña DEL Libro DE DANiel KahNEMAN: Pensar rápido, pensar despacio Thinking, Fast and Slow
}

\author{
Miguel Fernández Carrión \\ Escuela Normal Superior del Estado de México
}

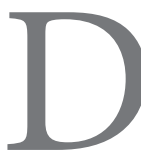
aniel Kahneman nace en Tel Aviv, se licencia en Psicología en la Universidad Hebrea de Jerusalén, tras servir cuatro años en el ejército israelí se doctora en 1961 en la Universidad de California en Berkeley. Desempeña la cátedra Eugene Higgins de Psicología de la Universidad de Princenton y asuntos públicos en la Escuela Woodrow Wilson de Asuntos Públicos e Internacionales. En 2002 obtiene el Premio Nobel de Economía, es por tanto el primer no economista a quien se le concede tal distinción, posteriormente también lo es Amartya Sen, entre otros, por su trabajo interdisciplinar pionero de integración de la psicología en las ciencias económicas, especialmente sobre lo con- cerniente al juicio humano y la toma de decisiones en entornos económicos de incertidumbre.

Su pensamiento teórico ha influido en áreas del conocimiento tan diversas como la economía, la medicina y la política.

En Pensar rápido, pensar despacio, Kahneman presenta el sistema de funcionamiento del pensamiento, que lo subdivide en dos modelos: el sistema uno, es rápido, intuitivo y emocional (el pensamiento rápido incluye las dos variantes del pensamiento intuitivo: el experto y el heurístico, así como las actividades mentales puramente automáticas de la percepción y la memoria), mientras que el sistema dos es 
más lento, deliberativo y lógico, de aquí el título del libro Pensar rápido, pensar despacio. En el momento de formular juicios y tomar decisiones personales se tiende a conjuntar el funcionamiento de los dos sistemas, tanto en la comprensión del impacto de la adversión a la pérdida y el exceso de confianza en las estrategias personales o empresariales, la dificultad de predecir lo que nos puede hacer felices en el futuro o el efecto de los sesgos cognitivos sobre todo lo que se hace.

Kahneman analiza el momento y el estado en el cual se debe confiar en las intuiciones y cuando no, y de qué modo se pueden aprovechar mejor los beneficios del pensamiento lento. Asimismo, propone enseñanzas prácticas sobre cómo tomar decisiones profesionales y personales, así como utilizar diferentes técnicas psicológicas para protegernos de los fallos mentales que nos crean problemas sociales.

El presente libro está conformado teóricamente con el "actual" concepto de los juicios personales y la toma de decisiones al respecto (elaborado por el autor en las últimas décadas) y las "ideas capitales" (originadas conjuntamente con Amos Tversky, "futura estrella" de la teoría de la decisión, en el Departamento de Psicologia de la Universidad Hebrea de Jerusalén, a partir de 1969, durante catorce años).

Amos pensaba con más lógica, con una orientación más teórica y un sentido infalible de la orientación. Yo era más intuitivo y estaba más centrado en la psicología de la percepción [...] Éramos lo bastante similares para entendernos uno a otro con facilidad, y lo bastante diferen- tes para sorprendernos mutuamente (Kahneman, 2013: 17).

El libro consta de cinco partes: la primera muestra los elementos básicos del tratamiento de los juicios y las eleciones de toma de decisión basadas en los dos sistemas (S): 1 (pensamiento rápido) y 2 (pensamiento lento). Propone la diferenciación entre las operaciones automáticas del S 1 y las operaciones controladas del S 2 y alude a la memoria asociativa (núcleo del $\mathrm{S}$ 1) que construye de forma continuada una interpretación "coherente" de lo que sucede en cada momento en el entorno del sujeto.

La segunda parte analiza la heurística de los juicios e intenta dar respuesta analíticamente a la interrogante ¿por qué nos resulta tan difícil pensar estadísticamente?, la respuesta viene dada porque el pensamiento asociativo, el metafórico y el casual está más generalizado, es más fácil. En cambio el estadístico "requiere pensar en muchas cosas a la vez, algo para lo que el Sistema 1 no está diseñado" (Kahneman, 2013: 27).

A partir de la consideración de las dificultades del pensamiento estadístico construye la tercera parte, en la cual se demuestra —según el autor- "nuestra excesiva confianza en lo que creemos saber y nuestra aparente incapacidad para reconocer las dimensiones de nuestra ignorancia y la incertidumbre del mundo en que vivimos" (Kahneman, 2013: 27).

En la cuarta parte sobre economía, trata de la naturaleza de la decisión y la "suposición de que los agentes económicos son racionales" (Kahneman, 2013: 27), pues existe una "desafortunada" tendencia a tra- 
tar de estudiar y dar solución a los problemas de forma aislada, y con "efectos marco, cuando las decisiones viene conformadas por características ilógicas instaladas en los problemas de elección" (Kahneman, 2013: 28), contraria a la racionalidad favorecida en la economía estándar.

La quinta parte se centra en el estudio de la distinción entre los "dos yo": el yo que experimenta cosas y el yo que las recuerda, "los cuales no tienen los mismos intereses" (Kahneman, 2013: 28). En este sentido Kahneman establece una diferenciación entre la utilidad experimentada y la utilidad de decisión, él indica: "los dos conceptos de utilidad coincidirían si las personas desean lo que les agrada y disfrutan de lo que eligen por ellas mismas, y esta suposición de una coincidencia va implícita en la idea general de que los agentes económicos son racionales" (Kahneman, 2013: 491), esto puede hacerse extensible al resto de elecciones que debe tomar el ser humano a lo largo de su vida.

La distinción entre dos yo es aplicada a la medición del bienestar, donde nuevamente encontramos que lo que hace feliz al yo que experimenta no es precisamente lo mismo que satisface al yo que recuerda [...] es algo que plantea algunas difíciles cuestiones, tanto para los individuos como para las sociedades que ven en el bienestar de la población un objetivo político (Kahneman, 2013: 28).

A modo de conclusión, presenta en orden inverso, las implicaciones de las tres distinciones realizadas a lo largo de los capítulos previos: entre el yo que experimenta y el yo que recuerda, entre el concepto de agente económico clásico y de la economía conductual, y entre el "automático" sistema 1 y el "esforzado" sistema 2.

Para terminar baste dar respuesta a la interrogante que establece el autor en relación con el contenido del libro:

¿Cómo podemos mejorar los juicios y las decisiones, los nuestros y los de las instituciones a las que servimos y que nos sirven a nosotros [esto es un eufemismo]? La respuesta es que poco podemos conseguir sin un esfuerzo considerable (...) La manera de bloquear los errores que origina el Sistema 1 es un principio sencillo: reconocer las señales de que estamos en un campo cognitivo minado, detenernos y pedir refuerzos al Sistema 2 (...) La voz de la razón puede ser mucho más tenue que la voz alta y clara de una intuición errónea y cuestionar nuestras intuiciones es poco agradable en medio de la tensión que acompaña a una gran decisión (...) La conclusión es que es mucho más fácil identificar un campo minado cuando vemos a otros caminando por él que cuando lo hacemos nosotros (Kahneman, 2013: 542-543).

A veces, no es tanto problema la toma de decisión como la reelaboración del problema planteado, aunque en ambos casos hay que aludir a la existencia de una serie de etapas en la producción de decisiones que - según el autor-, son: "el enmarque del problema que hay que resolver, la obtención de la información relevante previa a la decisión y la reflexión y revisión" (Kahneman, 2013: 544).

Al final del libro se reproduce el artículo conjunto de Tverky y Kahneman con 
el título de "El juicio bajo incertidumbre: heurísticas y sesgos", publicado originalmente en 1974 en la revista Science, vol. 185 y el elaborado igualmente por Kahneman y Tverky, en este orden de participación, sobre las "Elecciones, valores y marcos", presentado originalmente en el Congreso de la Asociación Estadounidense de Psicología en 1983 y publicado en American Psycologist, vol. 34, en 1984.

El libro Pensar rápido, pensar despacio, de Daniel Kahneman, es de interés; por una parte, porque sirve para ampliar la cultura general del lector instruido sobre estudios del pensamiento, así como del que está ávido de nuevos conocimientos, y por otra, porque puede aplicarse por igual a áreas del conocimiento de la medicina, la política y especialmente la economía. Por todo ello, es considerado como uno de los mejores libros de 2011 por The New York Times, The Economist, The Wall Street Journal y The Globe and Mail, pues cambiará "para siempre" la manera de pensar sobre cómo se piensa. De parecida forma se expresa The Economist, al señalar que "igual que Copérnico sacó a la Tierra del centro del universo y Darwin retiró al ser humano de su preeminencia biológica, Kahneman ha demostrado que no somos los pilares de sensatez que creemos ser" o como indica Wiliam Easterly, en Financial Times: "Hay muchos libros sobre la racionalidad y la irracionalidad humana, pero sólo una obra maestra: el libro de Daniel Kahneman".

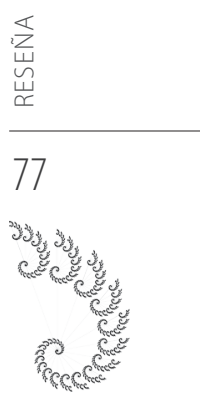

